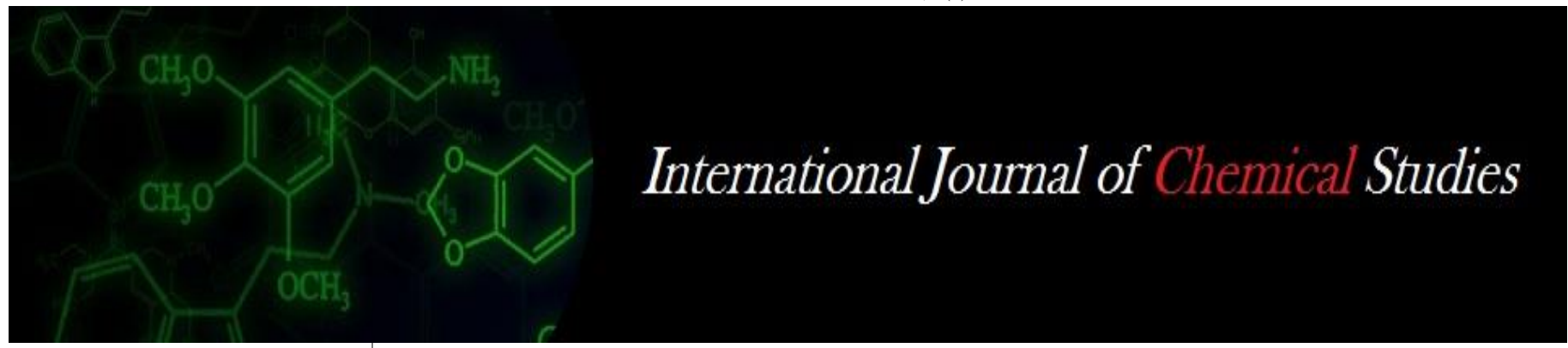

P-ISSN: 2349-8528

E-ISSN: 2321-4902

www.chemijournal.com

IJCS 2020; 8(2): 310-313

(C) 2020 IJCS

Received: 08-01-2020

Accepted: 12-02-2020

Anjali Jeena

Department of Veterinary

Medicine, C.V.A.Sc. GBPUAT,

Pantnagar, Uttarakhand, India

VS Rajora

Department of Veterinary

Medicine, C.V.A.Sc. GBPUAT,

Pantnagar, Uttarakhand, India

Niddhi Arora

Department of Veterinary

Medicine, C.V.A.Sc. GBPUAT,

Pantnagar, Uttarakhand, India

Surya Pratap Singh Chauhan

Department of Livestock

Production Management,

C.V.A.Sc. GBPUAT, Pantnagar,

Uttarakhand, India
Corresponding Author:

Anjali Jeena

Department of Veterinary

Medicine, C.V.A.Sc. GBPUAT,

Pantnagar, Uttarakhand, India

\section{Urinalysis in canine hepatic dysfunction: An important tool for clinical diagnosis}

\author{
Anjali Jeena, VS Rajora, Niddhi Arora and Surya Pratap Singh Chauhan
}

DOI: $\underline{\text { https://doi.org/10.22271/chemi.2020.v8.i2e.8785 }}$

\section{Abstract}

A clinico-pathological study was conductd on eighteen dogs screened for hepatic dysfunction. Urine samples were collected and processed for gross, microscopic and chemical examination. A low urine specific gravity as reported in these cases which is due to polyuria and polydipsia. The presence of bilirubinuria and bilirubin crystals in the urine was suggestive of canine hepatic dysfunction. An increment in urobilinogen was observed in the urine of dogs having compromised liver. Leucocytes were found in traces, ketonuria was observed in a fecases while there was no presence of nitrites.

Keywords: Liver, bilirubinuria, Urobilinogen, polyuria

\section{Introduction}

In veterinary medicine, hepatic failure has been defined as a sudden loss of more than $75 \%$ of functionally active hepatic mass. This leads to insufficient liver parenchyma which maintains synthetic and excretory requirements. The biochemical abnormalities and clinical signs of acute hepatic failure in canines include significant elevations in ALT liver enzyme activity and bilirubin concentration, loss of synthetic capabilities, coagulation abnormalities, and signs of hepatic encephalopathy, as seen in humans (Hughes et al., 1996) ${ }^{[9]}$. Many patients with hepatobiliary disease have polyuria and polydipsia and therefore a low urine specific gravity. Some dogs with PSVA have detectable ammonium biuratecrystalluria on urine sediment examination due to concurrent hyperuricaemia and hyperammonemia hence several examinations of fresh urine samples may be necessary in order to detect crystalluria. It is normal for some dogs (particularly male dogs) to have some conjugated bilirubin in their urine, but presence of hyperbilirubinuria is an indicative finding of excessive extravascular haemolysis or hepatobiliary disease (Santilli and Gerboni, 2003) ${ }^{[15]}$.

\section{Materials and methods}

\subsection{Sampling}

Canine patients were screened for hepatic dysfunction during the study period at Teaching Veterinary Clinical Complex, C.V.A.Sc, G.B.P.U.A\&T, Pantnagar, U.S. Nagar, Uttarakhand. Screening of dogs for hepatic dysfunction was based on the following criteria. On the basis of preliminary screening, 18 screened dogs affected with hepatic dysfunction were selected five $\mathrm{ml}$ of fresh urine sample was collected from each dog either at urination or by urinary catheter in a clean sterilized glass tube with preservative (Toluene) on day 0.

\subsection{Urine analysis}

The urine so collected was subjected to routine physical, chemical and microscopic examination. In the gross physical analysis, urine was evaluated for colour, odor, turbidity or any deposits. In the chemical analysis, urobilinogen, bilirubin, glucose, protein, ketones, specific gravity, erythrocytes, leucocytes, urine $\mathrm{pH}$ and bacteria were detected using dipstick method. For microscopic examination, centrifugation of urine samples was done at $2000 \mathrm{rpm}$ for 5 minutes. The supernatant was discarded and the sediments were thoroughly resuspended in the urine. A drop of the reconstituted sediment was transferred onto a microscopic glassslide and covered with glass slip. The quantity and the type of casts were assessed under low power (10X) of light microscope. 
High power (40X) was used to detect any abnormal structure. Slides were also examined for presence of crystals, microorganisms, urothelial cells, pus cells and erythrocytes.

\section{Results and Discussion}

Urine analysis is performed to obtain valuable information of urinary tract and body systems including liver. So, a complete urine analysis (dipstick, specific gravity, and sediment examination) is often done even though if one component part does not show any abnormality (Kumar et al., 2012) ${ }^{[10]}$. Considering frequency of urination, normal urination was reported in $6(33.33 \%)$ dogs, oligouria in $5(27.78 \%)$, polyuria in $4(22.22 \%)$, urinary incontinence in $2(11.11 \%)$ and urine retention in $1(5.56 \%)$. Polyuria/polydipsia could be because of hampered adrenal steroid metabolism, impaired portal vein osmoreceptor, renal medullary concentration gradient loss, encephalopathy (Hess and Bunch, 2000) [8] and loss of potassium (Bunch, 2003) ${ }^{[4]}$.

\subsection{Gross examination of urine in dogs with hepatic dysfunction}

Healthy dogs normally have acidic urine $\mathrm{pH}$ in nature. However, variations in urine $\mathrm{pH}$ can vary with the diet. Urine was observed in all the 18 positive screened dogs. Fifteen dogs $(83.33 \%)$ fell in the category of $\mathrm{pH}$ 5.5-7 and 3 dogs had $\mathrm{pH}>7$ as shown in table 1 . Out of 18 dogs, the colour of the urine was normal (straw coloured) in $8(44.45 \%)$ dogs, dark yellowish in $5(27.78 \%$ ) dogs and light (pale) yellowish in 2
(11.11\%) dogs. Transparent and reddish yellow urine each was observed in $1(5.56 \%)$ dog (Fig 1). Brownish coloured and greenish coloured urine each was observed in $1(5.56 \%)$ case. The pale coloured urine samples mostly contributes to a low specific gravity of <1.015 (Table 1). According to Forrester and Brandt (1994), the patients with dark red or brown urine may have haematuria, haemoglobinuria or myoglobinuria. Out of 18 dogs, the urine of $2(11.11 \%)$ dogs had ammoniac smell and $1(5.56 \%)$ dogs had a sweet fruity odour which was found to be due to presence of ketone bodies, whereas the urine of $15(83.33 \%)$ dogs had normal urineferousodour. Dogs which had revealed ammoniac odour were suffering from severe renal impairment, whereas dogs with sweet fruity odour had diabetic ketoacidosis (DKA). Macroscopically, the urine of $2(11.11 \%)$ dogs had turbidity whereas turbidity was absent in $16(18.89 \%)$ dogs. Turbidity of the urine could be attributed to the presence of crystals, casts or proteinuria. Urine specific gravity of the normal healthy dogs varies from 1.015-1.030. In the present study, the urine specific gravity was measured in 18 dogs and found to be less than 1.015 in $3(16.67 \%)$ dogs, more than 1.030 in 1 $(5.56 \%)$ dogs and rest of the $14(77.78 \%)$ dogs had urine specific gravity within normal range of 1.015-1.030 (Table 1). A low urine specific gravity is common in patient with liver disease due to polyuria and polydipsia (Bexfield and Watson, 2006; Chapman and Hostutler, 2013) [5, 2]. However, renal impairment associated with hepatic dysfunction also can contribute to the low urine specific gravity.

Table 1: Gross examination of urine in dogs with hepatic dysfunction $(n=18)$

\begin{tabular}{|c|c|c|}
\hline \multicolumn{2}{|c|}{ Gross examination of urine $(n=18)$} & Total $(n=18)$ \\
\hline \multirow{2}{*}{$\mathrm{Ph}$} & $5.5-7$ & $15(83.33 \%)$ \\
\hline & $>7$ & $3(16.67 \%)$ \\
\hline \multirow{6}{*}{ Colour } & Straw coloured & $8(44.45 \%)$ \\
\hline & Dark yellow & $5(27.78 \%)$ \\
\hline & Pale yellow & $2(11.11 \%)$ \\
\hline & Transparent & $1(5.56 \%)$ \\
\hline & Reddish yellow & $1(5.56 \%)$ \\
\hline & Greenish & $1(5.56 \%)$ \\
\hline \multirow{3}{*}{ Odour } & Ammoniac & $2(11.11 \%)$ \\
\hline & Fruity sweet & $1(5.56 \%)$ \\
\hline & Nil & $15(83.33 \%)$ \\
\hline \multirow{2}{*}{ Turbidity } & Present & $2(11.11 \%)$ \\
\hline & Absent & $16(88.89 \%)$ \\
\hline \multirow{3}{*}{ Specific gravity } & $<1.015$ & $3(16.67 \%)$ \\
\hline & $1.015-1.030$ & $15(83.33 \%)$ \\
\hline & $>1.030$ & $1(5.56 \%)$ \\
\hline \multirow{5}{*}{ Frequency of urination } & Normal urination & $6(33.33 \%)$ \\
\hline & Oliguria & $5(27.78 \%)$ \\
\hline & Polyuria & $4(22.22 \%)$ \\
\hline & Incontinence & $2(11.11 \%)$ \\
\hline & Retention & $1(5.56 \%)$ \\
\hline
\end{tabular}

Figures in parenthesis indicate percentage. (n) refer to number of dogs

\subsection{Routine and microscopic examination of urine}

Urine analysis provides information of urinary tract and other body systems including hepatic whereas the microscopic examination of urine is more accurate since many urine abnormalities might be present in animals with hepatic dysfunction (Bexfield and Watson, 2006; Kumar et al., 2012) ${ }^{[2,10]}$.Usually, a healthy dog's urine does not have erythrocytes however the presence of $1-2 \mathrm{RBC} / \mathrm{HPF}$ is generally not considered abnormal. In the urine of a healthy dog, up to five RBCs or pus cells per high power field (HPF) in the urine of dog are considered to be normal. In this study, out of 18 urine samples analysed, $2(11.11 \%)$ dogs had plenty of RBCs and pus cells count per HPF. The presence of pyuria and haematuria in the urine sediment reveals renal inflammation, haemorrhage or some infection in any part of urinary tract. Urothelial cells were observed in the urine of 4 (22.22\%) dog. Bilirubinuria was found in $10(55.56 \%)$ dogs among tested samples, but bilirubin crystals were reported only in 2 $(11.11 \%$ ) dogs with hyperbilirubinuria (Table 2, Fig 2). 
Table 2: Microscopic findings of urine in canines having hepatic dysfunction $(n=18)$.

\begin{tabular}{|c|c|}
\hline Observations & Number of dogs \\
\hline RBC & $2(11.11 \%)$ \\
\hline WBC & $3(16.67 \%)$ \\
\hline Urothelial cells & $4(22.22 \%)$ \\
\hline Bilirubinuria & $10(55.56 \%)$ \\
\hline Bilirubin crystals & $2(11.11 \%)$ \\
\hline Proteinuria & $3(16.67 \%)$ \\
\hline Bacteruria & $1(55.56 \%)$ \\
\hline Granular casts & $2(11.11 \%)$ \\
\hline Waxy casts & $1(55.55 \%)$ \\
\hline
\end{tabular}

Figures in parenthesis indicate percentage. (n) refers to number of dogs.

Bilirubinuria greater than $2+$ in a urine dipstick in a dog should head for suspicion of underlying hepatic dysfunction (Chapman and Hostutler, 2013) ${ }^{[5]}$. Granular casts were observed in $2(11.11 \%)$ dogs and waxy casts in $1(5.26 \%)$ dog. Proteinuria and bacteruria were detected in $3(16.67 \%)$ and 1 $(5.56 \%)$ dogs respectively (Table 2$)$. The granular casts are due to acute renal failure associated with acute hepatic failure, whereas waxy casts are sometimes occurring with chronic renal disease. Cystitis and pyelonephritis are characterized with bacterial pesence (Acierno and Senior, 2010) ${ }^{[1]}$. In this study, microscopic examination of urine was found to be more sensitive and reliable than dipstick test for examination of bilirubinuria which is highly suggestive of liver dysfunction.

\subsection{Chemical analysis of urine in dogs with hepatic dysfunction}

Complete chemical analysis of urine was performed on 18 fresh urine samples collected from dogs revealing signs of liver damage like jaundice and ascites beside elevation in liver enzymes (ALT and GGT) and abnormal changes in hepatic architecture based on ultrasonographic examination. There was a degree of variation in proteinuria in many of the cases. The degree of proteinuria was trace in 5(27.78\%), 1+ in $2(11.11 \%), 2+$ in $4(22.22 \%)$ and $3+$ in $7(38.89 \%)$ dogs (Table 3). Forrester (1997) observed that proteinuria was noted in Congestive Heart Failure, genital diseases, lower urinary tract infections (UTIs) and renal disorders. In this study, level of glucose was found in traces in $3(16.67 \%)$ dogs, $1+$ in $3(16.67 \%), 2+$ in $1(5.56 \%)$ and $3+$ in 2 $(11.11 \%)$. The remaining $9(50 \%)$ dogs showed negative glucosuria. Diabetes mellitus may contribute to the presence of glucose in urine. During chemical analysis of urine for the presence of blood $8(44.44 \%)$ dogs were found negative; blood cells to the degree of $1+$ were found in $3(16.67 \%)$ dogs, $2+$ in $2(11.11 \%)$ and $3+$ in $5(27.78 \%)$ dogs. Presence of blood in urine is mainly due to haematuria or haemoglobinuria. In this study bilirubin was found to be $1+$ in the urine of $2(11.11 \%)$ dogs which was associated with icterus, $2+$ in $3(16.67 \%)$ and $3+$ in $4(22.22 \%)$ dogs (Table $3)$. Bilirubinuria greater than $2+$ in a urine dipstick in a dog should raise the index of suspicion for underlying hepatic disease (Chapman and Hostutler, 2013) [5]. Increased bilirubinuria due to overspill is a finding in dogs with hyperbilirubinemia. Small quantities (traces) of conjugated bilirubin are found in the urine of normal dogs (particularly male dogs) due to the low renal threshold for bilirubin (Bexfield and Watson, 2012) ${ }^{[3]}$. The causes of increased bilirubinuria are therefore, an indicator of excessive extravascular haemolysis or hepatobiliary disease (Santilli and Gerboni, 2003) ${ }^{[15]}$. Nelson and Couto (1998) and Leib and Monroe (1997) observed that finding consistent in hepatobiliarydiseases on urine analysis includes bilirubinuria. In this study, bilirubinuria was never found in trace and was in excess concentration along with high rise of the specific liver enzymes and abnormal changes in the hepatic parenchyma on ultrasonography which indicates the hepatic origin of hyperbilirubinuria.

Measurement of urobilinogen by dipstick analysis has traditionally been used to assess the patency of the extrahepatic biliary system. Urine normally comprises of urobilinogen and its high amount is present along with hyperbilirubinuria and complete obstruction of bile duct. If there is complete obstruction of bile duct, no urobilinogen should be present in urine (uncommon in canines). In the present study, value of urobilinogen showed variation within the range of 0.1 to $8 \mathrm{mg} / \mathrm{dL}$. The concentration of urobilinogen was $0.1 \mathrm{mg} / \mathrm{dL}$ in $1(5.56 \%)$ urine samples, 0.2 $\mathrm{mg} / \mathrm{dL}$ in $11(61.11 \%)$ and $1 \mathrm{mg} / \mathrm{dL}$ in $3(16.67 \%)$ cases. Rest of dogs showed urobilinogen values of more than one. The values were $2 \mathrm{mg} / \mathrm{dL}$ in $1(5.56 \%)$ dogs, 4 as well as $8 \mathrm{mg} / \mathrm{dL}$ each was observed in $1(5.56 \%)$ urine sample (Table 3$)$. After haemolysis, there is increment in urobilinogen in the urine and the dogs having hepatic dysfunction can develop clinical and laboratory evidence of renal tubular dysfunction (Langlois et al., 2013) ${ }^{[11]}$.

Table 3: Chemical analysis of urine in dogs with hepatic dysfunction $(n=18)$.

\begin{tabular}{|c|c|c|}
\hline Parameter & Result & Total \\
\hline \multirow{5}{*}{ Glucose (mg/dl) } & Negative & $9(50 \%)$ \\
\hline & Trace & $3(16.67 \%)$ \\
\hline & +1 & $3(16.67 \%)$ \\
\hline & +2 & $1(5.56 \%)$ \\
\hline & +3 & $2(11.11 \%)$ \\
\hline \multirow{4}{*}{ Blood } & Negative & $8(44.44 \%)$ \\
\hline & $1+$ & $3(16.67 \%)$ \\
\hline & $2+$ & $2(11.11 \%)$ \\
\hline & $3+$ & $5(27.78 \%)$ \\
\hline \multirow{3}{*}{ Protein (mg/dl) } & Trace & $5(27.78 \%)$ \\
\hline & $1+$ & $2(11.11 \%)$ \\
\hline & $2+$ & $4(22.22 \%)$ \\
\hline
\end{tabular}




\begin{tabular}{|c|c|c|}
\hline & $3+$ & $7(38.89 \%)$ \\
\hline \multirow{4}{*}{ Bilirubin (mg/dl) } & Negative & $9(50 \%)$ \\
\hline & $1+$ & $2(11.11 \%)$ \\
\hline & $2+$ & $3(16.67 \%)$ \\
\hline & $3+$ & $4(22.22 \%)$ \\
\hline \multirow{5}{*}{ Leucocytes } & Trace & $1(5.56 \%)$ \\
\hline & 1 & $2(11.11 \%)$ \\
\hline & 2 & $1(5.56 \%)$ \\
\hline & 3 & $2(11.11 \%)$ \\
\hline & Negative & $12(66.67 \%)$ \\
\hline \multirow{2}{*}{ Ketone bodies (mg/dl) } & Present & $2(11.11 \%)$ \\
\hline & Absent & $16(88.89 \%)$ \\
\hline \multirow{6}{*}{ Urobilinogen (mg/dl) } & 0.1 & $1(5.56 \%)$ \\
\hline & 0.2 & $11(61.11 \%)$ \\
\hline & 1 & $3(16.67 \%)$ \\
\hline & 2 & $1(5.56 \%)$ \\
\hline & 4 & $1(5.56 \%)$ \\
\hline & 8 & $1(5.56 \%)$ \\
\hline
\end{tabular}

Figures in parenthesis indicate percentage. (n) refer to number of dogs.

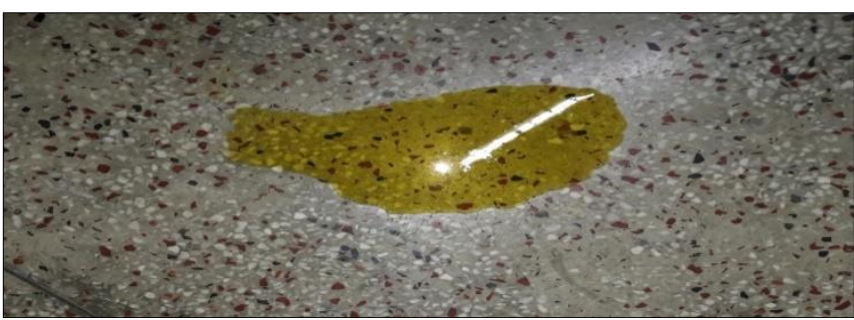

Fig 1: Dark yellow cloured urine by a liver compromised dog

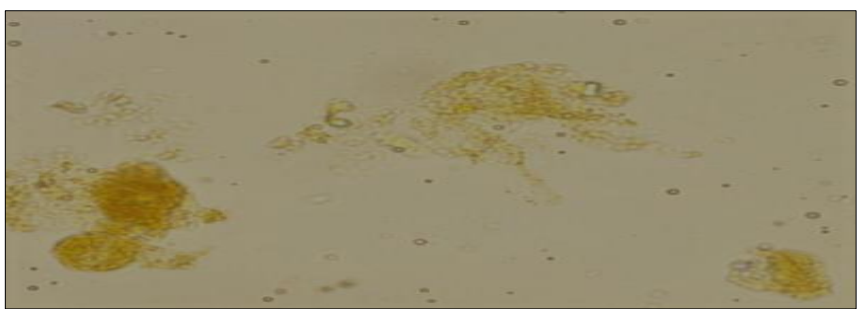

Fig 2: Bilirubin crystals on microscopic examination of urine

\section{References}

1. Acierno MJ, Senior DF. Urinary disorders. In: Schaer M. Clinical Medicine of the Dog and Cat. Manson publishing Ltd, Alfred Place, London, 2010, 505-560.

2. Bexfield N, Watson P. Diagnosis of canine liver disease. In Pract.2006; 28:444-453.

3. Bexfield NH, Buxton RJ, Vicek TJ, Day MJ, Bailey SM, Haugland SP et al. Breed, age and gender distribution of dogs with chronic hepatitis in the United Kingdom. Vet. J.2012; 193(1):124-8.

4. Bunch SE. Diagnostic tests for the hepatobiliary system. Nelson R W. Couto C G, eds. Small Ani. Inter. Med.2003; 3:488.

5. Chapman SE, Hostutler RA. A Laboratory Diagnostic Approach to Hepatobiliary Disease in Small Animals. Veterinary Clinic of Small Animal. 2013; 43(6):1209-25.

6. Forrester SD. Diseases of kidney and ureter. In: Practical Small Animal Internal Medicine (Eds.) Leib MS and W E Monroe. W B Saunders CO., Philadelphia, 1997.

7. Forrester SD, Brandt KS. The diagnostic approach to the patient with acute renal failure. Vet. Med.1994; 212(89):214-18.

8. Hess PR, Bunch SE. Diagnostic approach to hepatobiliary diseases. In: Kirk's Current Vety. TherapySmall Ani. Pract. W. B. Saunders And Co. Philadelphia.2000; 13(4):659-63.
9. Hughes D, Moreau RE, Overall KL. Acute hepatic necrosis and liver failure associated with benzodiazepine therapy in six cats 1986-1995. J. Vet. Emer. Critical Care, 1996; 6(1):13-20.

10. Kumar V, Kumar A, Varshney AC, Tyagi SP, KanwarMS, Sharma SK. Diagnostic imaging of canine hepatobiliary affections: a review. Vet. Med. Inter, 2012, 672107.

11. Langlois DK, Smedley RC, Schall WD, Kruger JM. Acquired proximal renal tubular dysfunction in 9 Labrador Retrievers with copper-associated hepatitis (2006-2012). J. Vetey. Inter. Medi.2013; 27(3):491-9.

12. Langlois DK, Smedley RC, Schall WD, Kruger JM. Acquired proximal renal tubular dysfunction in 9 Labrador Retrievers with copper-associated hepatitis (2006-2012). J. Vetey. Inter. Medi.2013; 27(3):491-9.

13. Leib M, Monroe. Practical of Small Animal Internal Medicine, W.B. Saunders CO., Philadelphia, 1997.

14. Nelson RW, Couto CG. Diagnostic Tests For The Hepatobiliary System, In Small Ani. Inter. Med.Mosby, Inc., Misssouri. 1998; 2:487-509.

15. Santilli RA,Gerboni G. Diagnostic imaging of congenital portosystemic shunts in dogs and cats: a review. The Vet. J.2003; 166:7-18. 